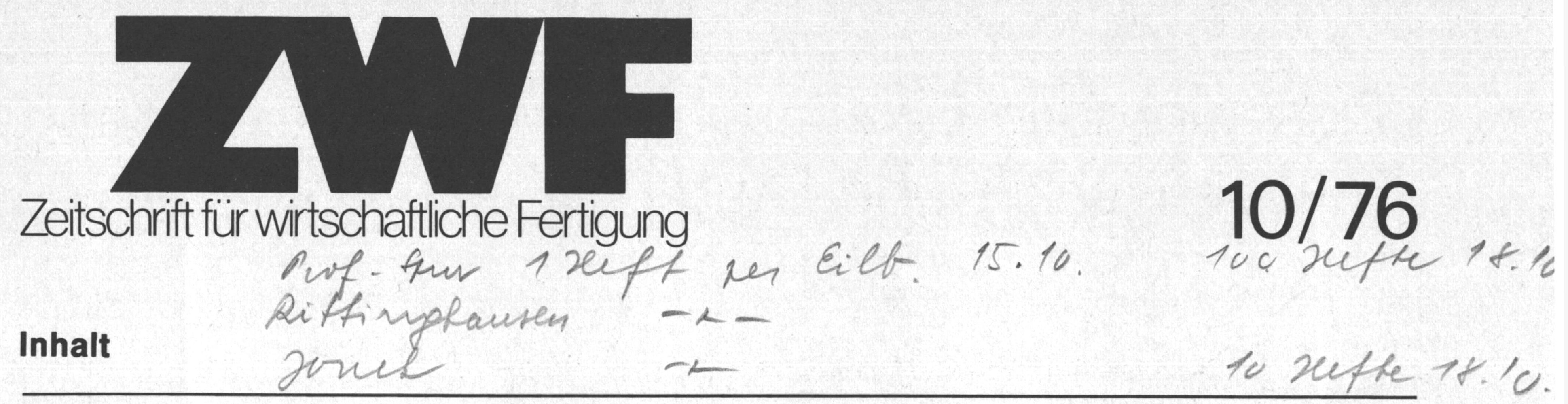

\section{Akzente und Tendenzen}

\section{Fertigungsplanung und Fabrikeinrichtungen}

R. Schaumann, Die Verschleißgröße am Werkzeug, seine Abhängigkeit von der Schneidkeilgeometrie und der Werkstoff-Schneidstoff-Paarung

Drehmoment-Meßwertaufnehmer zur Kontrolle der Schneidleistung

Entscheidungshilfe für die Auswahl von Gabelstaplern

U. Dworak und V. Gomoll, Wirtschaftlicher Einsatz von Schneidkeramik

Seminar-Automatisierte Konstruktion und Fertigung . . . 425

E. Altmann, Weiterentwicklungen bei Präzisionswerk-

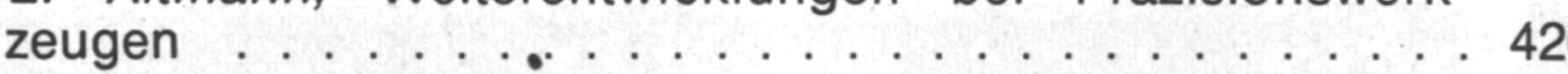

H. Hauri, Präzisionswerkzeuge zur rationellen Bohrungsbearbeitung

IMTO-76

H. Widman, Qualitäts- und Leistungsprüfung von Diamantnadel-Abrichtwerkzeugen

Schweißtechnisches Kolloquium ... . . . . . . . . . 440

CNC Blechbearbeitungszentren ............ 440

$R$. Ichimiya und $U$. Heisel, Neue Möglichkeiten der Kompensation thermischer Störeinflüsse an Werkzeugmaschinen

Neuer Vierkanal-A/D-Wandler zum direkten Rechneran-

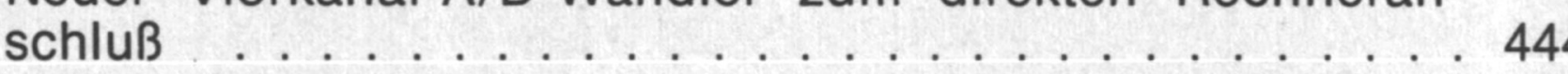

\section{ZwF-Lehrgang}

G. Spur, Spanende Werkzeugmaschinen II-4. Thermisches Verhalten ..................

413

\section{Dokumentation}

G. Koschnick, Beschichtete und keramische Schneidstoffe

\section{Wärmebehandlungstechnik}

D. Dengel und W. Kassner, Statische und dynamische Härteprüfung mit dem Scherkraft-Härteprüfer ERNST . . . . . . 454

Kassettenregistrierung von analogen und digitalen Meßdaten

E. Schreiber, Härterisse und Schleifrisse - Ursachen und Auswirkungen von Eigenspannungen (Teil1) . . . . . . 460

Kantenschleifmaschine ................ . 465

Standzeitvergleich mit nitrid-beschichteten Wendeplatten . 465

\section{Neues aus der Industrie}

100 Jahre Werkzeugmaschinenfabrik Heyligenstaedt \& Co. . 466 Wärme dämmen im Temperaturbereich bis $1800^{\circ} \mathrm{C}$. . . . . 466

Forschungsreport ................ 466

Koordinaten-Schleifkopf ............. 466

Diamant-Räumahle . . . . . . . . . . . . . . 467

Pneumatisch feinverstellbare Bohrstange . . . . . . . . . 467

Wendeplattenfräser . . . . . . . . . . . . . . . . 467

Konturparalleles Entgraten von Löchern in gewölbten Ober-

flächen ................... . . 467

Schneiden-Einstellgerät mit Werkzeugaufnahmen-Ma-

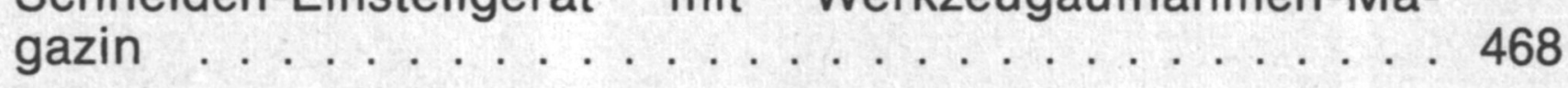

Maschinentisch für schwere Montageaufgaben . . . . . . 468

\title{
Zur Titelanzeige
}

\section{MH 2000 NC}

Universal-Genauigkeits-Bohr- und Fräswerk

Fräsen, bohren, ausdrehen und natürlich zentrieren, reiben, senken, gewindeschneiden usw. usw. mit der Genauigkeit eines Bohrwerkes, mit der Leistungsfähigkeit einer Fräsmaschine, sowohl mit der Horizontal- als auch mit der Vertikalspindel (natürlich auch schräg bei geschwenktem Senkrechtfräskopf), an quaderförmigen Werkstücken, an Prismen, ja in jedem auf einer Kugelkalotte möglichen Winkel, das sind die Kennzeichen dieser wahrhaft universalen Bearbeitungsmaschine für den Maschinenund Fahrzeugbau, für den Formenbau, für den Werkzeug- und Vorrichtungsbau und nicht zuletzt für die komplexen und geometrisch anspruchsvollen Fertigungsprobleme der Luft- und Raumfahrttechnik. Gleichgültig wo der Schwerpunkt des Einsatzes liegt: Werkstück aufsetzen und in einer Aufspannung fertigbearbeiten.

\section{MAHO}

Werkzeugmaschinenbau

Babel \& Co.

D-8962 Pfronten,

West Germany

Telefon: (0 83 63) 89-1

Telex: 05-41414

Foto: Gordian Eberle, BFF Pfronten

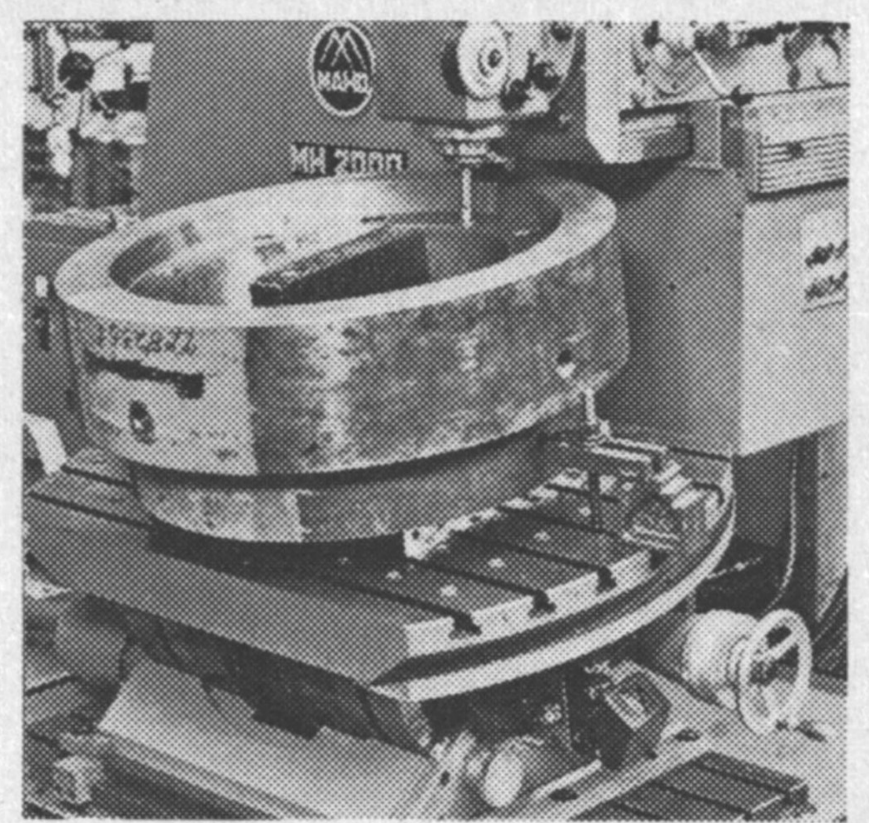




\section{Das einmalige Nachschlagewerk für Praxis, Wissenschaft und Studium!}

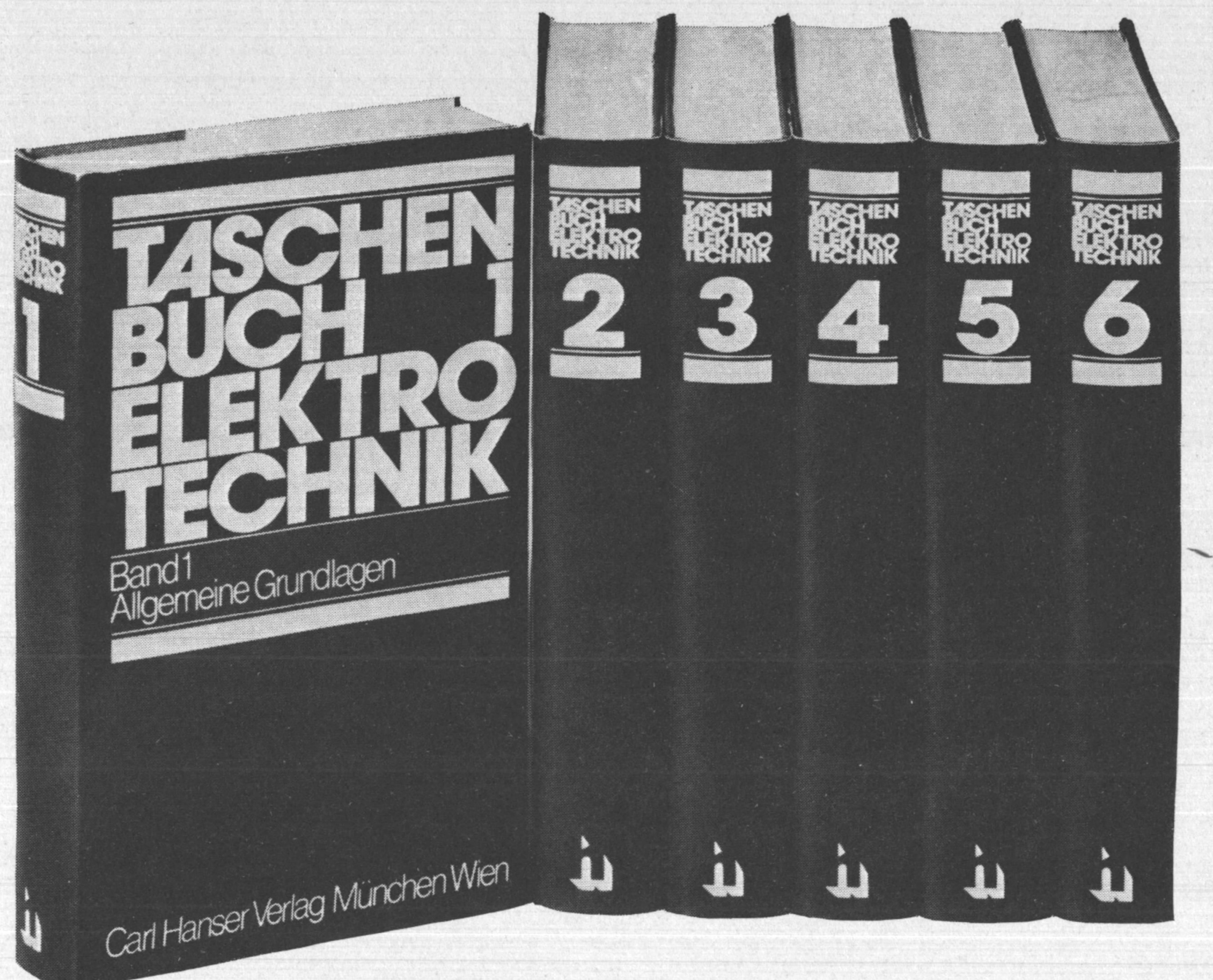

Jetzt zum günstigen Subskriptionspreis
Herausgegeben von Prof. Dr. sc. techn. Eugen Philippow.

Neue, völlig überarbeitete Ausgabe in 6 Bänden. Jetzt mit SI-Einheiten und DIN-Normen!

Band 1

Allgemeine Grundlagen

Band 2

Grundlagen der Informationstechnik

\section{Band 3}

Bauelemente und Funktionseinheiten der Informationstechnik

\section{Band 4}

Systeme der Informationstechnik

\section{Band 5}

Elemente und Baugruppen der Elektronenenergietechnik

\section{Band 6}

Systeme der Elektrotechnik, Isoliertechnik, Energieumwandlung

Das "Taschenbuch Elektrotechnik" ist hochgeschätzt und eines der erfolgreichsten Nachschlagewerke überhaupt. Mit Recht, denn dieses "Taschenbuch" bringt in konzentrierter Form das gesamte Basiswissen, die grundsätzlichen Begriffe und Bezeichnungen der gesamten Elektrotechnik: klar formuliert, systematisch und übersichtlich geordnet sowie ergänzt und vertieft durch technische Daten und Erfahrungswerte in Gestalt von Tafeln, Diagrammen, Zeichnungen und Übersichten.
Der Benutzer dieses exzellenten Nachschlagewerkes erhält damit exakte, leicht greifbare und rasch verwertbare Informationen und Unterlagen, wie sie bei der Bearbeitung theoretischer und praktischer Aufgaben der Elektrotechnik benötigt werden. Ableitungen sind nach Möglichkeit vermieden worden und erscheinen nur dann, wenn sie für die richtigen Anwendungen von Formeln usw. von Bedeutung sind. Diese Neuausgabe des berühmten Werkes verwendet konsequent die gesetzlichen SI-Einheiten.

Das ,'Taschenbuch Elektrotechnik' ist für jeden Ingenieur und Techniniker der Praxis ebenso unentbehrlich wie für Wissenschaftler, Dozenten und Studenten, denn es läßt wirlich keine Frage offen und bietet wirkungsvolle Hilfe bei allen wissenschaftlichen und praktischen Arbeiten.

\section{Band 1}

Allgemeine Grundlagen

896 Seiten, 625 Bilder, 166 Tafeln. 1976. Gebunden 58 ,- DM, bei Subskription auf das 6-bändige Gesamtwerk nur 48,- DM.

\section{Inhaltsübersicht}

Theoretische Grundlagen der Elektrotechnik

Mathematik und spezielle Rechenverfahren der Elektrotechnik

Elektrophysik

Elektrische Meßtechnik

Werkstoffe der Elektrotechnik

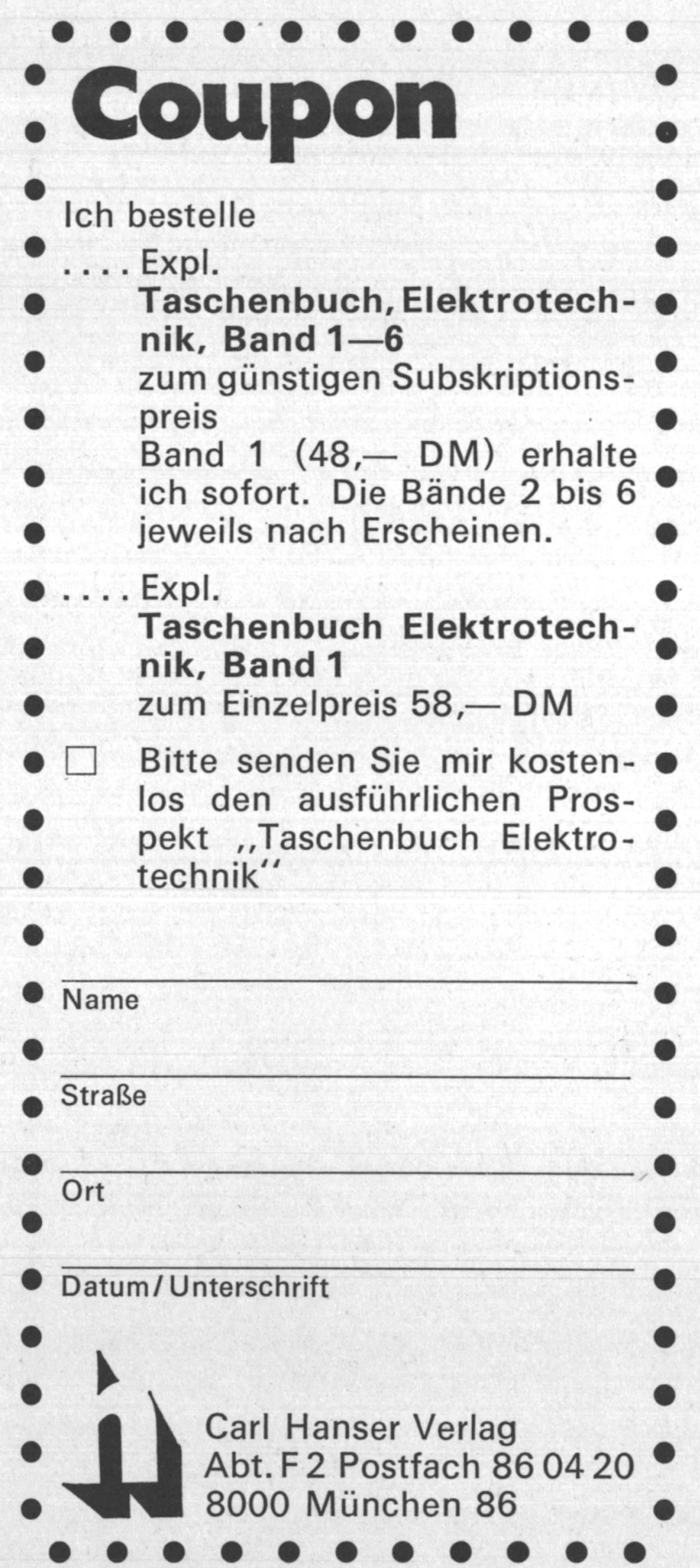

